\title{
8 Knights Problem \& Extension to Fractals
}

\author{
Shreyan Mondal \\ (Department of Engineering Physics, Delhi Technological University (formerly Delhi College of Engineering), \\ New Delhi)
}

\begin{abstract}
The paper aims to develop solutions from a pre-determined solution to the 8 knight's problem with the aid of linear transformation termed as' Shifting Techniques'. The term 'Shifting Technique' implies displacement of the position of the two knights while maintaining a definite number of gaps between them. The employment of: 'Vertical, Horizontal, Alternate, Diagonal \& L-shaped shifting techniques has been effectively generated limited number of solutions to the 8 knights problem due to symmetric pattern constraints.. The use of symmetry \& parallelism illustrates the generation of missing solutions on the board. Further cases devised in the paper have been discussed to imbibe the reader with the concept of 'Shifting Techniques'. The latter parts of the paper discuss the applications of these shifting techniques in the generation of fractals that are an important class in image development. These have been extensively used in image construction. Figure Solutions generated via MATLAB coding at the end of the paper elucidates the generation of solutions to illustrate the idea of fractal generation
\end{abstract}

\section{Introduction}

The ' 8 Knights 'problem traces its roots from the famous ' 8 queens' problem that has baffled mathematicians and theorists since centuries pertaining to its unique solutions and complexity. The problem aims to place 8 knights on a chess board in such a way that each row/column is occupied by a single knight and no knight removes the other one from the chess board. Employment of different shifting techniques namely: Vertical, Horizontal, Alternate, Diagonal \& L shaped have led to solution generation. Therefore no distinction between unique and distinct solutions has been made. Devised formulae used throughout the paper shall guide the reader about the shifting techniques/transformation on the board. The application of symmetry and the use of parallelism has been extensively used to generate solutions.

\section{Shifting Strategies}

5 shifting techniques have been employed to generate solutions namely: [1],[2]

$\begin{array}{ll}\text { - } & \text { Vertical } \\ \text { - } & \text { Horizonta } \\ \text { - } & \text { Diagonal } \\ \text { L-shaped }\end{array}$

Under the vertical shifting technique the knights are shifted vertically along the columns while maintaining an odd displacement between them. Using (1), for $\mathrm{N}=6$, a total of 4 repetitions have to be performed to obtain the original configuration. Similarly the below transformations can be generated for the cases $\mathrm{N}=2 \& 4$.

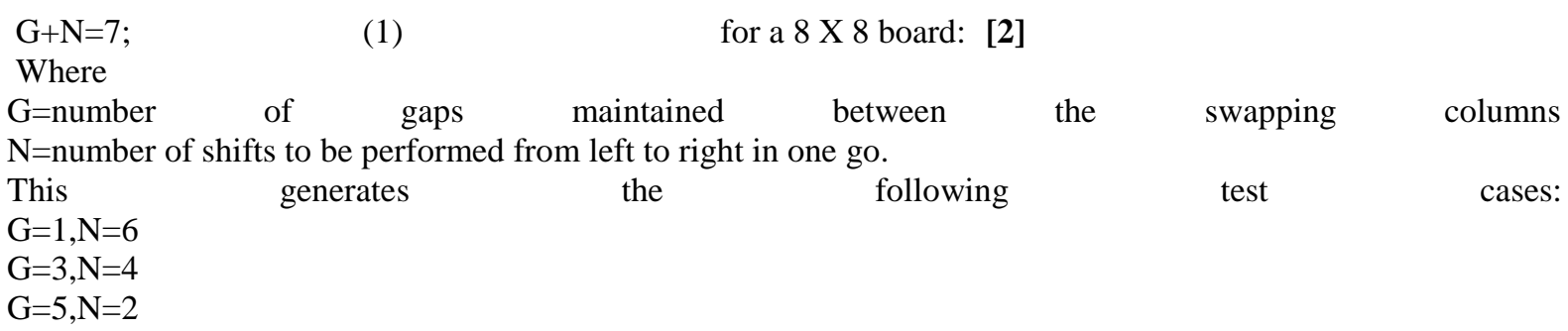

From above cases $\mathrm{G}$ will always be odd and $\mathrm{N}$ will be even. If $\mathrm{G}$ is even, the shifting techniques lead to a no solution case. Usually the value of $\mathrm{G}$ is known in the equations while the value of $\mathrm{N}$ is determined from (1). A more generalized form is given below. For a $\mathrm{K} \mathrm{X} \mathrm{K}$ board, the relation becomes:-

$\mathrm{G}+\mathrm{N}=(\mathrm{K}-1)$

[3]

This holds true only for 2-dimensional systems. 


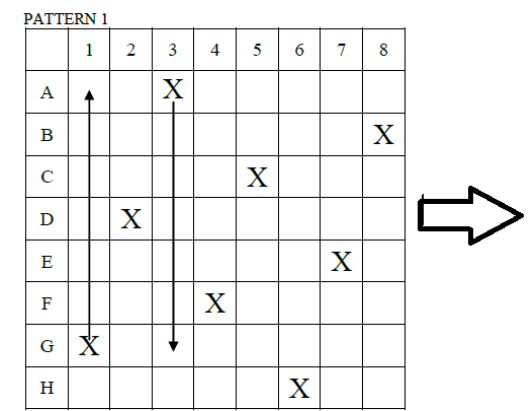

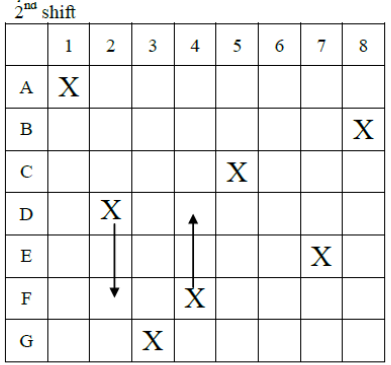

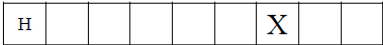

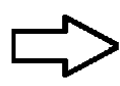
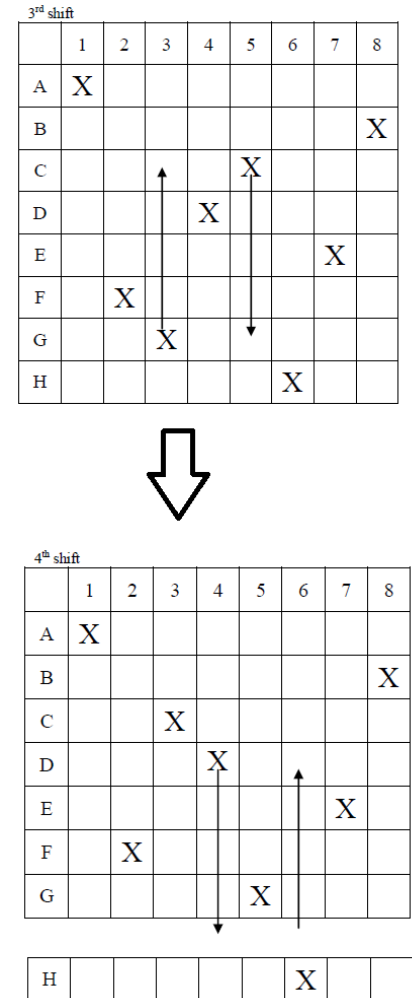

Fig1.

Table1 illustrates the corresponding value of $\mathrm{N} \& \mathrm{G}$ and the repetitions that have to be performed to obtain the original configuration.

Table1.

\begin{tabular}{|c|c|c|}
\hline Value of N & Value of G & Repetitions \\
\hline 6 & 1 & $6 * 4=24$ \\
\hline 4 & 3 & $4 * 2=8$ \\
\hline 2 & 5 & $2 * 2=4$ \\
\hline
\end{tabular}

The Alternate technique involves the usage of horizontal and vertical technique simultaneously while following their conditions for shifting. Here both the techniques horizontal and vertical are either performed in clockwise or anti-clockwise direction. Even though this technique is more cumbersome than the former, the number of moves to obtain the required solution is minimized. TABLE 2. Below summarizes the movement of the knights on the board in an Alternate technique when a gap of $\mathrm{N}=1$ is maintained for both the rows and columns. An equal gap $(\mathrm{G}=1,3$ or 5$)$ is always maintained for both the vertical and horizontal shifts. Here a vertical shift $\&$ a horizontal shift is performed corresponding to an odd move $\&$ an even move respectively.

Table2.

\begin{tabular}{|l|c|l|c|}
\hline $\begin{array}{l}\text { Move } \\
\text { number }\end{array}$ & $\begin{array}{l}\text { Type of } \\
\text { shift }\end{array}$ & $\begin{array}{l}\text { Move } \\
\text { number }\end{array}$ & $\begin{array}{l}\text { Type of } \\
\text { shift }\end{array}$ \\
\hline 1 & Vertical & 7 & Vertical \\
\hline 2 & Horizontal & 8 & Horizontal \\
\hline 3 & Vertical & 9 & Vertical \\
\hline 4 & Horizontal & 10 & Horizontal \\
\hline 5 & Vertical & 11 & Vertical \\
\hline 6 & Horizontal & 12 & Horizontal \\
\hline
\end{tabular}

Diagonal technique involves the displacement of the knights in an upward or downward direction either leftwards or rightwards by a fixed displacement value ' $n$ '. Say for instance if one of the knights is moved in an upward left direction by 4 chebyshev distances [4], so are the other knights. Displacement by a fixed chebyshev 
distance leads to generation of solution from the existing one. However it is not true for all configuration of the chess board.

\begin{tabular}{|c|c|c|c|c|c|c|c|c|}
\hline & 1 & 2 & 3 & 4 & 5 & 6 & 7 & 8 \\
\hline A & & & $X$ & & & & $x$ & \\
\hline B & & & & $\mathbf{x}$ & & & & $X$ \\
\hline C & $x$ & & & & $\mathrm{X}$ & & & \\
\hline D & & $X$ & & & & $x$ & & \\
\hline E & & & $x$ & & & & $X$ & \\
\hline $\mathrm{F}$ & & & & $\mathrm{X}$ & & & & $\mathbf{x}$ \\
\hline G & $X$ & & & & $x_{n}$ & & & \\
\hline H & & $\mathbf{x}$ & & & & $X$ & & \\
\hline
\end{tabular}

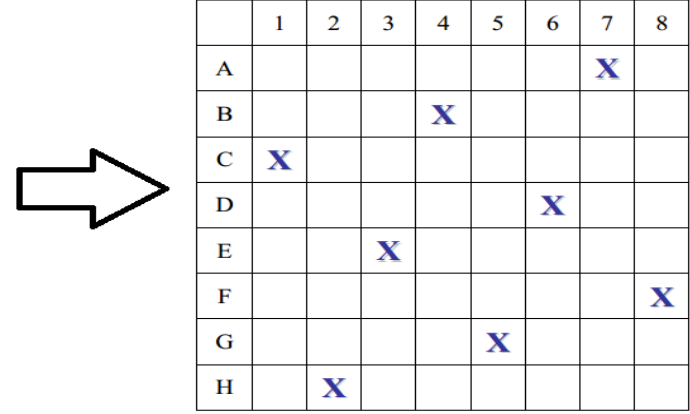

Fig2.

L-Shaped technique involves the transformation of knights in such a manner that the 4 outer knights are displaced in a single move while the inner most knights are displaced to their adjacent positions in 3 moves for a given configuration (as shown in Fig3(b)) of the chess board. Fig3(a). Illustrates this movement. If the inner knights are displaced in a clockwise direction, so are the outermost knights. Both the inner and outer knights follow suit. The types of movements are summarized in TABLE 3.

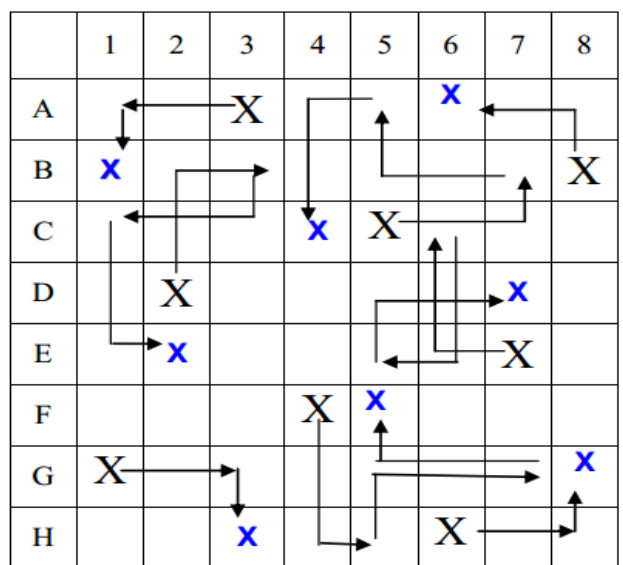

Fig3.(a)

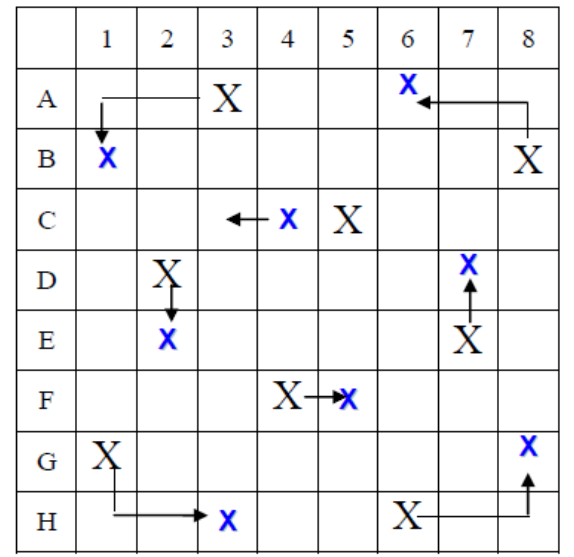

Fig3(b).

\begin{tabular}{|l|l|c|c|}
\hline & \multicolumn{2}{|c|}{ Type of displacement } & $\begin{array}{c}\text { Number of } \\
\text { moves }\end{array}$ \\
\hline $\begin{array}{l}\text { Inner } \\
\text { Knights }\end{array}$ & Clockwise & $\begin{array}{c}\text { Anti- } \\
\text { clockwise }\end{array}$ & 3 \\
\hline $\begin{array}{l}\text { Outer } \\
\text { knights }\end{array}$ & Clockwise & $\begin{array}{c}\text { Anti- } \\
\text { clockwise }\end{array}$ & 1 \\
\hline
\end{tabular}

Table3.

Specific Cases

Certain cases where given solutions are missing and are required to be generated from the original pattern are discussed below.

$1^{\text {st }}$ case

- Generation of Solutions using 3 Non-Collinear Knights: If two knights are collinear (minimum 3 knights on the board) then a line is drawn through the 2 collinear knights. A line is drawn through the non-collinear knights such that it is parallel to the former line drawn.

- The number of squares between the two former lines is determined. It will always be odd, equal to ' $n$ '.

- At the $((n+1) / 2)$ th square a line is drawn such that it is parallel to the line drawn through the original noncollinear knights. The $1^{\text {st }}$ missing knights occupies this $((n+1) / 2)$ th square.

- Through the $1^{\text {st }}$ missing knight a line is drawn intersecting both the lines drawn earlier.

- The $2^{\text {nd }} \& 3^{\text {rd }}$ knights occupy the position where the line drawn in the previous step intersects the two parallel lines. 
- A line is drawn through the $1^{\text {st }}$ non-collinear knight through which no line passes. This line is parallel to the line passing through the $((\mathrm{n}+1) / 2)$ th square. This line drawn will intersect the line passing through the original non-collinear knight. This is the $4^{\text {th }}$ missing knight. The position of the $5^{\text {th }}$ missing knight is determined by analogy.

- The missing knights are represented by the pink dots.

\begin{tabular}{|c|c|c|c|c|c|c|c|c|}
\hline & 1 & 2 & 3 & 4 & 5 & 6 & 7 & 8 \\
\hline A & & & $x$ & & & & & \\
\hline B & & & & & & & & \\
\hline C & & & & & 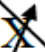 & & & \\
\hline D & & $X$ & & & & & & \\
\hline E & & & & & & & & \\
\hline $\mathrm{F}$ & & & & $\lambda$ & & & & \\
\hline G & & & & & & & & \\
\hline $\mathrm{H}$ & & & $\checkmark$ & & & & & \\
\hline
\end{tabular}

$2^{\text {nd }}$ case

Fig4(a)

- Generation of solutions using 3 collinear knights: In this case a line is drawn through the 3 non-collinear knights.

- Lines are drawn through each of the knights such that these are parallel to one another. The position of the last square is determined where these lines end their trace. The number of squares between any of the knights and the last square where the line leaves its trace is determined.

- If number of squares is odd, the $1^{\text {st }}$ missing knight occupies the last position where the line ends its trace.

- If the number of squares is even the knight occupies just before the last trace of the line.

- If the line extends over $m$ squares the $3^{\text {rd }}$ missing knights occupies the $((m+1) / 2)$ the position.

- A line is drawn through the positions of the $1^{\text {st }}$ and the $2^{\text {nd missing }}$ knights. The number squares between them is determined.

- $\quad$ The $4^{\text {th }}$ missing knight is placed at the $m$ th cell from the $2^{\text {nd }}$ missing knight.

- The position of the $5^{\text {th }}$ missing knight is again determined via analogy.

- The missing knights are represented by the blue dots.

\begin{tabular}{|c|c|c|c|c|c|c|c|c|}
\hline & 1 & 2 & 3 & 4 & 5 & 6 & 7 & 8 \\
\hline A & & & $X$ & & & & & \\
\hline B & & & & & & & & \\
\hline $\mathrm{C}$ & & & & & & & & \\
\hline D & & $X$ & & & & & 1 & \\
\hline $\mathrm{E}$ & & & & & & & 2 & \\
\hline F & & & & & & & & 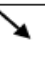 \\
\hline G & $x^{*}$ & & & & & & & \\
\hline $\mathrm{H}$ & & & & & & & & \\
\hline
\end{tabular}

Fig4(b) 

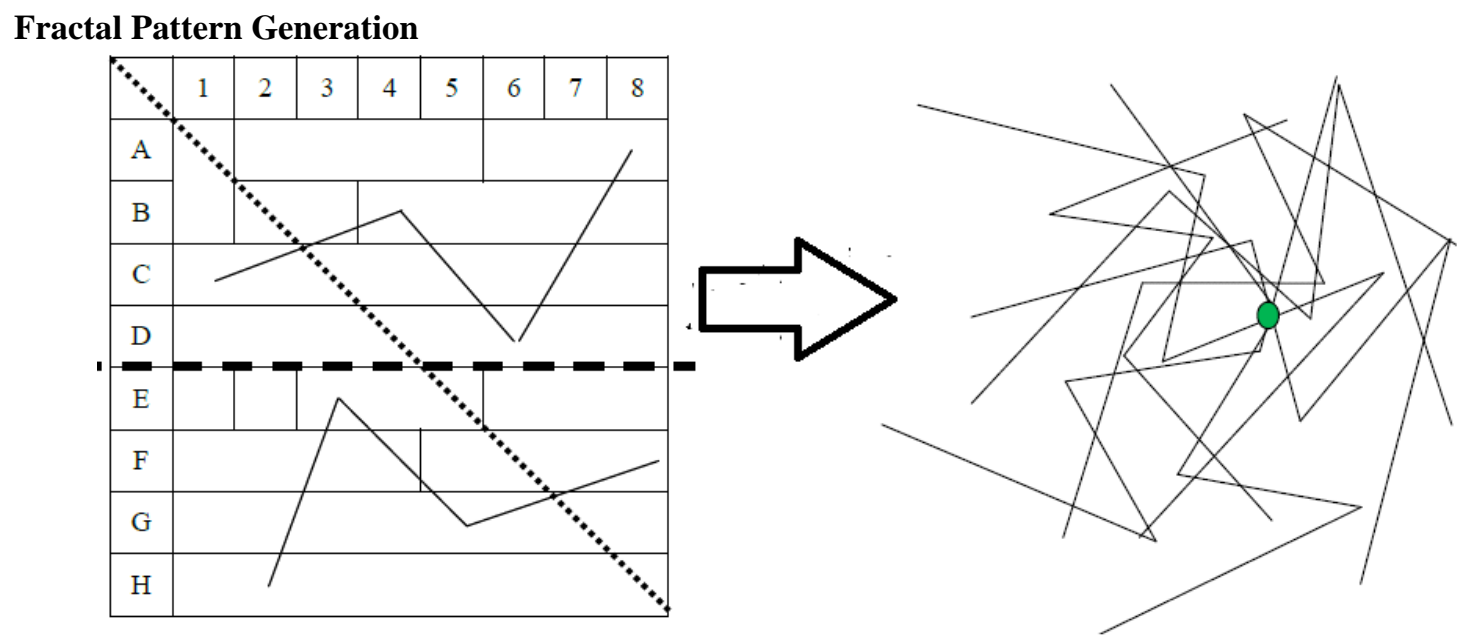

Fig.5

To geometrically construct a deterministic (non-random) self-similar pattern, we start with a given geometric shape called the initiator. Subparts of the initiator are then replaced with a pattern called the generator. Consider the transformation shown in fig3. Revolving this zigzag pattern about the thickened dashed line and also about the diagonal a new pattern can be obtained where the singular pattern has been rotated by theta degrees (0-360). The green dot at the center represents the center about which the image is revolved. This is one of the basic principles of image/pattern generation using fractals. Symmetrical \& super-imposable fractals about a line or an axis are used to replace the distorted/missing portions of image or a pattern. [5]. The figure below shows the pattern created by a solution to the 8 knight problem that can be used to construct design over a $4 \mathrm{X} 4$ region.

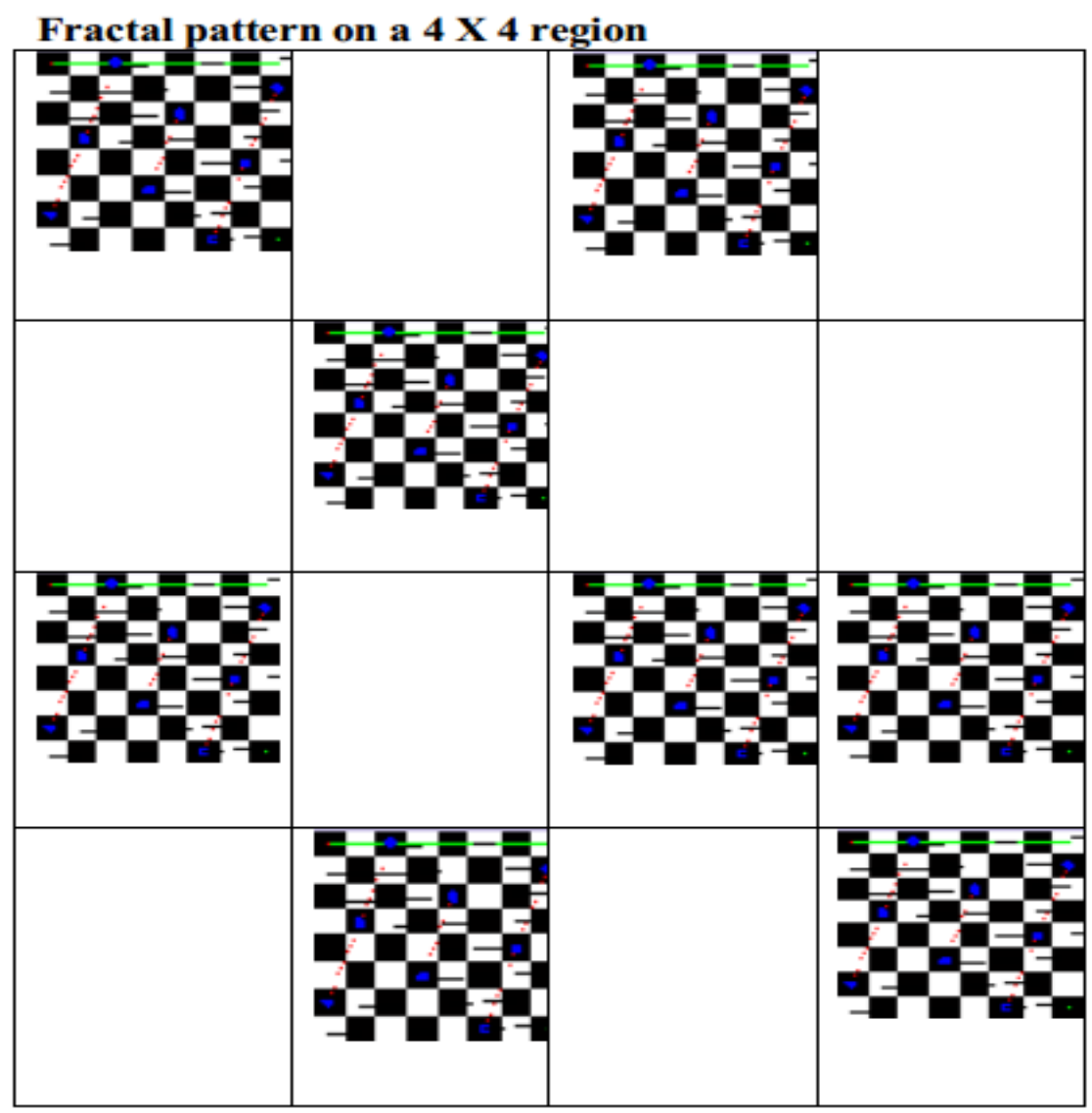

Fig6. 


\section{Generated Solution using MATLAB}

Fig6. Shows the solution generated to the 8 knight'sproblem. This is the simplest solution to the 8 knight's problem [6]. The solutions are represented by blue colored dots while the red lines denote their path or solution lines.

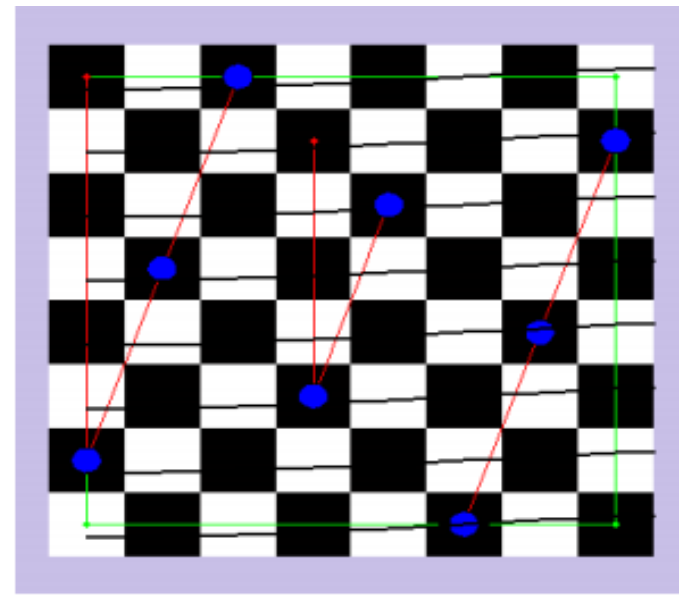

Fig7.

Fig8. illustrates the magnified view of the solution to the 8 knight's problem. In fig7. The green lines indicate a boundary tracing of the chess board into various symmetric sections for convenience. The blue dots in the figure below satisfy all the conditions of the 8 knight's problem. Also the red lines satisfy conditions for parallelism and symmetry and follow the shifting techniques employed to generate the solutions. It may be noted that all dots that are a solution to the 8 knight's problem always lie on the squares of the same colors (in this case but not always). The shorter red line in the middle is equidistant from both the longer lines.

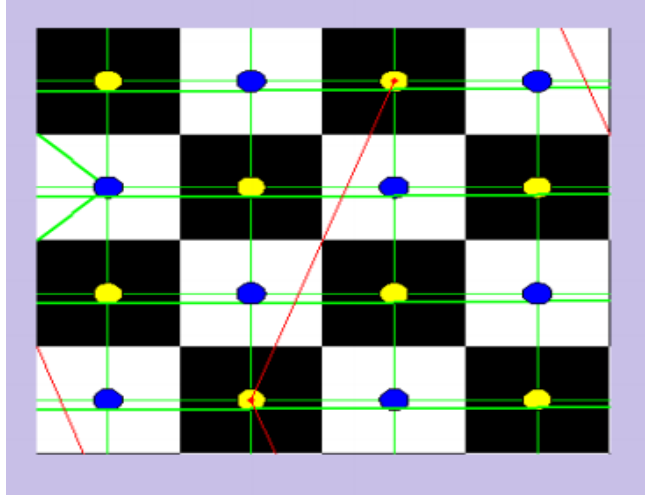

Fig8.

\section{Extension of Principle}

The pattern created by the 8 knight's problem on the chess board can be considered as the building block of an image. An image of dimensions $4 \mathrm{~N}$ X $4 \mathrm{~N}$ can be sub-divided into $\mathrm{N}$ blocks across the rows and columns to give $4 \mathrm{X} 4$ image. If a solution arrangement of the 8 knights problem is used to build a fractal set in an image, it can be replicated over the remaining (N. $\left.{ }^{\wedge} 2\right)-1$ blocks to create a symmetric pattern all over the board. In the figure below the entire pattern is repeated in two squares. It can be extended to the entire $4 \mathrm{~N} X$ $4 \mathrm{~N}$ grid to obtain the required pattern. Reiteration of blue dots along with the red lines makes the 8 knights pattern appear like fractal on a given flooring structure or mosaic. Only 8 out of the 16 squares contain the pattern while the remaining ones are kept empty for convenience.

\section{Acknowledgements}

I would like to acknowledge the concentrated and relentless efforts of my mentor Dr. Nilam without whom the completion of this paper would have been unmanageable. Considering the fact that the stated references have assisted me in the development of this paper, I would also like to attribute my grueling sessions of self-experimentation and verification of chess techniques that have responsibly molded the earlier parts of the paper. The usage of MATLAB coding is an attempt to elucidate the concept of fractal generation to create symmetrical patterns. 


\section{Conclusion}

Hence with the employment of shifting techniques we have generated solutions to the 8 knight's problem from a pre-existing one which in turn can be generated via backtracking method. A greater success has been owed to the vertical and horizontal shifting techniques in generating solutions. As stated earlier the alternate technique is more cumbersome since it leads to confusion in the arrangement of the knights on the board while the diagonal and L-shaped moves are applicable only for specific configurations. The problem does not aim in tallying the total number of solutions to the 8 knight's problem but in generating solutions from a preexisting one. Different solutions generated have been used to construct fractals which form an important class of image development. Linear transformations (shifting techniques) have addressed the main target of image construction via fractals \& the principles involved have been further extended.

\section{References}

[1]. Realization of 8 queens problem(www.linkspringer.com)

[2]. Self Experimentation of chess techniques.

[3]. Self Experimentation of Game Shifting Techniques.

[4]. Permutation Arrays under Chebyshev distance, Information Theory Te Tsung Lin,; Shi-Chun Sai ; Wen-Guen-Zeng ,IEEE Transactions

[5]. Fractal Geometry methods

[6]. Image Processing techniques in MATLAB (www.stackoverflow.com) 